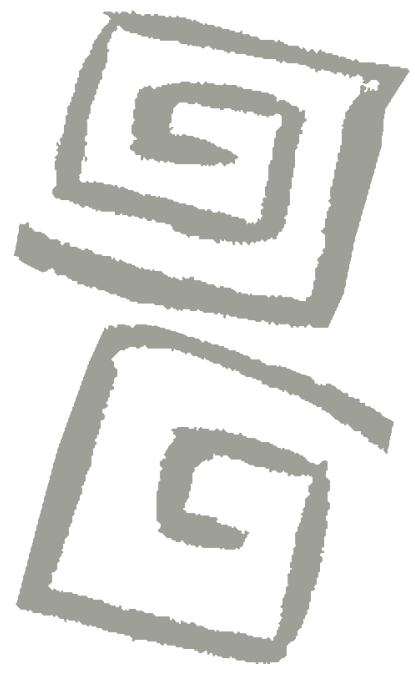

Stela Nazareth Meneghel', Rita de Cássia Maciazeki-Comes², Montserrat Sagot ${ }^{3}$, Betty Ruth Lozano Lerma ${ }^{4}$, Fernanda Souza de Bairros ${ }^{5}$, Aline Blaya Martins ${ }^{6}$

\section{Mujeres conversando con mujeres: un encuentro en el seminario Rutas Críticas 8}

\author{
Women talking to women: a meeting on \\ Critical Routes 8
}

${ }^{1}$ Médica. Doctora en Medicina. Coordinadora Programa de Pós-Graduação em Saúde Coletiva, Universidade Federal do Rio Grande do Sul, Rio Grande do Sul, Brasil. $\bowtie$ iD

${ }^{2}$ Psicóloga. Doctora en Psicología. Profesora, Programa de Pós-Graduação em Psicología, Universidade Federal do Rio Grande, Rio Grande do Sul, Brasil. $\square$ (iD

${ }^{3}$ Socióloga. Doctora en Sociología. Centro de Investigación en Estudios de la Mujer (CIEM), Universidad de Costa Rica, San José, Costa Rica. $\bowtie$ (iD)

${ }^{4}$ Socióloga. Doctora en Estudios Culturales Latinoamericanos. Directora de investigaciones, profesora Unibautista, Cali, Colombia. $\triangle$ (iD)

${ }^{5}$ Nutricionista. Doctora en Epidemiología. Profesora, Programa de Pós-Graduação em Saúde Coletiva, Universidade Federal do Rio Grande do Sul, Rio Grande do Sul, Brasil. $\square$ (iD)

${ }^{6}$ Dentista. Doctora en Odontología. Profesora, Programa de Pós-Graduação em Saúde Coletiva,

Universidade Federal do Rio Grande do Sul, Rio Grande do Sul, Brasil. $\square$ (iD)
RESUMEN Los seminarios "Rutas Críticas", realizados desde 2006, tienen por objetivo discutir y presentar datos de investigaciones e intervenciones sobre violencia contra la mujer. Este texto presenta una síntesis narrativa del Seminario Internacional Rutas Críticas 8, denominado "Feminicidios: necesitamos hablar de eso", realizado en Porto Alegre, en 2017. Se utilizó el formato de entrevista para presentar las narrativas, referenciales teóricos y situaciones particulares de cada región. Los feminicidios aumentaran bajo el modelo neoliberal que está ocurriendo en los países de América Latina y hay una sobremortalidad de mujeres jóvenes, migrantes, sin redes de protección, pobres y racializadas. La publicación de síntesis como esta constituye una oportunidad de hacer públicos los datos de investigaciones que incluyen la denuncia de situaciones específicas de riesgo y vulnerabilidad. El artículo comparte la reflexión crítica y las conversaciones entre investigadoras brasileñas e hispanoamericanas, buscando potencializar acciones de enfrentamiento a las violencias.

PALABRAS CLAVES Violencia contra la Mujer; Violencia de Género; Poblaciones Vulnerables; América Latina.

\begin{abstract}
The "Critical Routes" seminars, held since 2006, aim to discuss and present data on research and interventions regarding violence against women. This text presents a narrative synthesis of the International Seminar on Critical Routes 8, entitled "Feminicides: We need to talk about this," held in Porto Alegre in 2017. The interview format was used to present the narratives, theoretical references and specific situations of each region. Feminicides have increased under the neoliberal model that is taking place in the countries of Latin America, and there is an excess mortality among young, migrant, unprotected, poor and racialized women. The publication of these types of syntheses is an opportunity to make public research data that denounce specific situations of risk and vulnerability. The article shares critical reflections and conversations among Brazilian and Spanish-speaking Latin American researchers, seeking to strengthen actions to combat violence.

KEY WORDS Violence Against Women; Gender-Based Violence; Vulnerable Populations; Latin America.
\end{abstract}




\section{EMPEZANDO LA CONVERSACIÓN}

En Porto Alegre, del 28 al 29 de septiembre de 2017, tuvo lugar el Seminario Internacional Rutas Críticas 8. La temática de los feminicidios, asesinatos de mujeres motivados por la condición de género fue el enfoque dado a esta octava edición. Las Rutas Críticas son seminarios temáticos enfocados en la violencia contra la mujer, iniciados en 2006. Fueron ocho seminarios, algunos con alcance internacional y con apoyo de agencias de fomento brasileñas: el Conselho Nacional de Desenvolvimento Científico e Tecnológico (CNPq), la Coordenação de Aperfeiçoamento de Pessoal de Nivel Superior (CAPES), el Ministerio de la Salud, la Secretaría de las Políticas para las Mujeres, la Universidad Federal de Rio Grande do Sul (UFRGS) y Red Unida. Los temas versaron sobre las rutas críticas recorridas por mujeres en situación de violencia, las herramientas para enfrentar las violencias, la transversalidad de género en las políticas públicas, los feminicidios, la equidad y las desigualdades de género. Estos encuentros se realizaron en varias ciudades brasileñas $y, a$ partir de ellos, se organizaron dos libros ${ }^{(1,2)}$, diversos textos en la revista Athenea digital ${ }^{(3,4)}$, además de artículos en revistas científicas sobre los temas abordados en los eventos ${ }^{(5,6,7)}$.

El acceso a los seminarios era abierto y gratuito y en cada uno de ellos participaron más de 100 personas, estudiantes, trabajadores de la salud, de las ciencias sociales, jurídicas y militantes de movimientos sociales. Los seminarios propiciaron el espacio para la divulgación de investigaciones y para denunciar las inequidades perpetradas contra las mujeres, validadas por la comunidad científica, la militancia feminista y la red de servicios que combaten las violencias, respondiendo a una demanda de educación permanente para fortalecer las políticas públicas destinadas a las mujeres.

El Seminario Internacional Rutas Críticas 8 fue organizado e impartido por mujeres que trabajan en universidades latinoamericanas, en redes de investigación que abordan los temas vinculados a los estudios de género, involucradas en actividades de docencia, investigación y extensión. Entre las seis participantes de esta conversación, Montserrat Sagot es socióloga, docente e investigadora de la Universidad de Costa Rica, donde coordina el Centro de Investigación en Estudios de la Mujer (CIEM), además de ser la coordinadora para Costa Rica de la Encuesta Internacional de Violencia Contra las Mujeres; Betty Ruth Lozano Lerma, socióloga y doctora en Estudios Latinoamericanos, es directora de Investigaciones y profesora de la Unibautista en Cali, Colombia; Stela Nazareth Meneghel, Aline Blaya Martins y Fernanda Souza de Bairros son profesoras e investigadoras de la Universidad Federal de Rio Grande do Sul, en el área de salud colectiva, y desarrollan investigaciones en los temas de las violencias en relación con las mujeres, el género, las políticas públicas y la población negra. Rita de Cássia Maciazeki-Gomes, es psicóloga, profesora e investigadora de la Universidad Federal de Rio Grande y de la Universidad Federal de Rio Grande do Sul, en el área de salud Colectiva y psicología. Todas ellas desarrollan actividades de extensión y son militantes feministas que actúan junto a mujeres en situación de violencias (Montserrat y Stela), políticas públicas para mujeres (Aline), mujeres campesinas y procesos de subjetivación (Rita) y movimiento negro (Betty y Fernanda).

Presentamos aquí una síntesis narrativa $^{(8,9)}$ de las afecciones provocadas por nuestra participación en las Rutas Críticas, que siguen reverberando en nuestras reflexiones, conversaciones y prácticas cotidianas, con el fin de seguir la discusión y convocar a otras tantas personas, incluso aquellas que no estuvieron presentes.

\section{Rutas Críticas: la fuerza de una conexión latinoamericana en la lucha contra las violencias}

Rita: ¿Cómo surgió la idea de realizar los seminarios Rutas Críticas?

Stela: Los seminarios "Rutas Críticas" se iniciaron con una investigación que se inspiró en 
un estudio desarrollado por la Organización Panamericana de la Salud (OPS), denominado "La Ruta Crítica de las mujeres afectadas por la violencia intrafamiliar en América Latina"(10), coordinado por la investigadora Montserrat Sagot Rodríguez, de la Universidad de Costa Rica. Se denomina rutas críticas al itinerario seguido por las mujeres, que empieza con la decisión y la determinación de hacer cesar la violencia intrafamiliar y apropiarse de sus vidas y las de sus hijos. La ruta crítica es un proceso que se construye a partir de las decisiones y acciones emprendidas por las mujeres para tratar de terminar con la violencia y las respuestas encontradas tanto en su ámbito familiar y comunal, así como en el institucional. La investigación sobre las rutas críticas de mujeres en situación de violencia acompaña el camino de estas mujeres por las instituciones para conocer los factores que las impulsan a buscar ayuda y las dificultades para llevar adelante tal decisión, además de los significados sobre la violencia entre el personal de los servicios que deben ofrecer respuestas a este problema de salud pública. La investigación Ruta Crítica fue desarrollada en el marco del Proyecto Fortalecimiento y Organización de Mujeres y Acciones Coordinadas entre el Estado y Sociedad Civil para la Prevención y Atención de la Violencia Intrafamiliar, coordinado por el Programa Mujer, Salud y Desarrollo de la OPS y se constituye en una herramienta para la atención integral a la violencia intrafamiliar. El hecho de que Brasil no haya participado en esta investigación nos motivó a proponer una investigación similar y a organizar los dos primeros seminarios para discutir la ruta crítica enfrentada por las mujeres brasileñas. Una tercera edición se realizó en 2011, en Porto Alegre, en la Universidade Federal do Rio Grande do Sul, que se enfocó en las situaciones límite de la violencia, incluyendo asesinatos de mujeres y de cuerpos feminizados motivados por el género.

La cuarta edición ocurrió en 2012, en Río de Janeiro, a partir de la asociación entre la Universidad de Costa Rica y la Universidade Federal do Rio Grande do Sul, en el marco del $10^{\circ}$ Congreso Internacional de la
Red Unida, y se discutió el género en el ámbito de las políticas públicas y experiencias exitosas en el enfrentamiento de las violencias. El recrudecimiento de formas graves de violencia de género nos llevó a proponer una quinta edición, en 2013, que se enfocó en los femicidios que, en aquella época, eran un tema poco discutido en Brasil, pero presente en la agenda de América Latina y el Caribe. El evento se alineó a la discusión que ocurría en Brasil, coordinada por la Secretaría de Políticas para las Mujeres. El sexto seminario se realizó en 2014, en Fortaleza, en el marco del $11^{\circ}$ Congreso de la Red Unida, y tuvo como tema la equidad en el día a día de la prestación de servicios en la atención básica en salud. La séptima edición se realizó en la ciudad de Campo Grande, en Mato Grosso del Sur, durante el $12^{\circ}$ Congreso Internacional de la Red Unida y se discutió la desigualdad de género y sus efectos sobre la salud y el bienestar de las mujeres. En todo momento contribuyeron las y los investigadores asociados provenientes de países como Costa Rica, Honduras, Argentina, Colombia, y también de Haití, España e Italia, además de las y los trabajadores de instituciones brasileñas.

\section{Feminicidios: hay que hablar de eso...}

Rita: ¿Por qué fue elegido el tema feminicidios en la octava edición del seminario?

Stela: El concepto de femicidio es relativamente reciente, fue formulado por Diana Russel en $1976^{(11)}$ ante un Tribunal Internacional de Derechos Humanos. Para Russel, los femicidios (femicide en inglés) son asesinatos de mujeres por el hecho de ser mujeres, o sea, motivados por cuestiones de desigualdad de género. Es decir, el concepto de femicidio hace referencia al asesinato misógino de mujeres por parte de hombres de sus familias, parejas o exparejas, o atacantes sexuales -conocidos o desconocidos-, cuando los cuerpos de las mujeres son cosificados, usados como trofeos, como instrumento de reivindicación del "honor" o de venganza entre hombres. El femicidio expresa de forma 
dramática la desigualdad de relaciones entre lo femenino y lo masculino y muestra una manifestación extrema de dominio, terror, vulnerabilidad social, de exterminio e, incluso, de impunidad. La incorporación y el uso del concepto generó nuevos cuestionamientos, y autoras como Marcela Lagarde ${ }^{(12)}$ diferencian femicidio, o asesinato misógino de mujeres, de feminicidio, o asesinato de mujeres pautado en género en contextos de negligencia del Estado e impunidad con relación a esas muertes. Sin embargo, esta distinción hecha por Lagarde es cuestionada por varias autoras, incluyendo la misma Diana Russell, ya que mueve el eje central de la problemática del femicidio/feminicidio de la misoginia y la desigualdad de género a la impunidad y la respuesta del Estado. Al tratarse de un concepto relativamente nuevo, el debate continuó y hubo países que optaron por utilizar en la tipificación legal el término femicidio, mientras que otros optaron por el uso de feminicidio, ambos para designar el asesinato misógino de las mujeres. Brasil optó por el uso del concepto feminicidio en la reciente tipificación legal. Por este motivo, nos alineamos a esta denominación ${ }^{(5)}$.

Las mujeres fueron subyugadas durante toda la historia de la humanidad, violadas y muertas en tiempos de guerra, intercambiadas o vendidas en tiempos de paz. Esto sucedió en la conquista de las Américas, una historia de genocidio y asimilación forzada, afectando a las mujeres indígenas y las mujeres traídas de África. Más recientemente, los países hispanoamericanos, en contextos de super explotación producida por el neoliberalismo, se convirtieron en regiones extremadamente violentas para las mujeres, presentando las tasas más altas de feminicidios a escala mundial, acompañadas por explotación sexual, prostitución forzada, tráfico, genocidio de mujeres pertenecientes a grupos o etnias minoritarias, prácticas perpetradas por grupos mafiosos y paramilitares. En estos escenarios, cientos de mujeres mexicanas desaparecieron y murieron con tortura, crueldad y violencia sexual. Estos crímenes ocurrieron (y ocurren) en la frontera México-EEUU y alcanzaron, en su mayoría, a jóvenes trabajadoras de "maquilas" (empresas transnacionales ubicadas en estas áreas de "libre" comercio), y a migrantes racializadas, procedentes de varias regiones de Centroamérica que, atraídas por los empleos, se trasladan solas y sin redes de protección. Los feminicidios en Centroamérica se agudizaron en los contextos del capitalismo salvaje que se instaló en estos territorios. Las organizaciones criminales, como pandillas, mafias y maras, proliferan bajo la complacencia del Estado, de agentes jurídicos, policiales y políticos que usan a las mujeres como "víctimas sacrificiales" en una demostración necrófila de poder y de fuerza ${ }^{(13)}$.

En los diversos recortes territoriales de investigaciones que realizamos en la última década ${ }^{(14,15,16)}$ se observó una sobremortalidad de mujeres negras brasileñas en diferentes contextos territoriales. Estos asesinatos incluyen ejecuciones, muchas de las cuales se realizan en territorios ocupados por el narcotráfico. Estas muertes no despiertan empatía en la población y no son consideradas feminicidios, porque ese crimen aún se ve como consecuencia de la violencia doméstica en la intimidad de las parejas. En Porto Alegre, los datos de las encuestas policiales del período 2006-2010 muestran que los feminicidios son, en gran parte, ejecuciones en lugares donde la violencia urbana o estructural es elevada ${ }^{(17)}$. En Colombia se están desalojando poblaciones negras e indígenas de territorios ocupados desde el período colonial, para implementar megaproyectos de interés transnacional. Para someter y desterritorializar estas poblaciones, grupos armados al servicio del capital atemorizan, persiguen y eliminan a los miembros más frágiles y los índices de feminicidios han aumentado vertiginosamente ${ }^{(6,18)}$. Hay que subrayar que el riesgo no es igual para todas, los feminicidios tienen color y clase social y las más vulnerables son las jóvenes, migrantes, indígenas, negras, sin redes de protección, y que ejercen ocupaciones estigmatizadas como la prostitución. Esta situación, más el aumento del conservadurismo en la sociedad actual, que vulnerabiliza sobre todo a las mujeres, nos ha llevado a elegir el tema de feminicidios en esta octava edición. 
Rita: ¿Y por qué plantear la temática de los feminicidios racializados?

Fernanda: En Brasil, el mapa de la violen$\mathrm{Cia}^{(19)}$ indica que las muertes por homicidio de mujeres negras aumentó en la última década, mientras que los homicidios de mujeres blancas disminuyeron. Por lo tanto, las discusiones sobre el feminicidio deben tener en cuenta la cuestión racial. En este seminario participó Isabel Clavelin, periodista y militante feminista, con larga trayectoria en el Movimiento de Mujeres Negras, que a partir de la lectura de Sueli Carneiro ${ }^{(20)}$ mostró cómo todos los tipos de violencias -físicas, psicológicas, sexuales, patrimoniales y letales- fueron una constante en este país contra las mujeres negras, pero se escondieron bajo el mito de la democracia racial. Pensar en el feminicidio de mujeres negras significa recordar el tráfico transatlántico y visibilizar las torturas, las violaciones en el periodo colonial e históricamente sufridas por las mujeres negras. El racismo es determinante para que esas violencias, infracciones de derechos y feminicidios sean perpetrados contra las mujeres negras y para la naturalización y banalización de estas muertes. Pensar el racismo es traer el malestar del mito de la igualdad racial, que oculta las vidas prematuramente perdidas de mujeres negras. En este momento, necesitamos hablar de los feminicidios, desde la perspectiva de las mujeres negras, pobres y silenciadas históricamente.

\section{Del fascismo social al recrudecimiento de las violencias}

Aline: ¿Cuáles son los determinantes de esas muertes en nuestra sociedad?

Montserrat: La violencia contra las mujeres es reconocida como un problema social de grandes dimensiones, producto de una organización social estructurada sobre la base de la desigualdad. No creemos más que eso sea un problema personal, de malas relaciones, generado por la pobreza, sino un problema ocasionado por la desigualdad social. La violencia es una manifestación extrema de discriminación, un arma letal para mantener la subordinación de las mujeres y de los cuerpos feminizados. Los escenarios de las violencias contra los hombres y contra las mujeres son completamente diferentes: entre el $60 \%$ y el $70 \%$ de los homicidios de mujeres son cometidos por hombres cercanos y por razones sexuales, mientras que menos del $8 \%$ de los asesinatos de hombres son cometidos por razones sexuales y por mujeres cercanas. Los hombres son asesinados debido a la violencia criminal o por asuntos del mundo público. Los feminicidios son la forma más extrema de violencia de género practicada contra las mujeres, realizada por hombres para tener dominio y control sobre ellas ${ }^{(11)}$.

Necesitamos pensar el género en relación con otras categorías sociales como clase social, etnia, edad, orientación sexual, con las que el género se construye y opera. Los feminicidios ocurren cuando hay una lógica de desigualdad de poder entre los sexos y el cuerpo marcado de una mujer violada y tratada como un objeto es un indicador de esta desigualdad. Por eso es necesario entender el contexto, los motivos del asesinato y cómo y dónde el cuerpo fue descartado. El feminicidio expresa así la desigualdad entre lo masculino y lo femenino y la relación de dominio, de terror, de vulnerabilidad social y, en muchos casos, de impunidad. Las causas no se encuentran en las características personales de los perpetradores, sino en el estatus social de hombres y mujeres. Los cuerpos de las mujeres se convierten en la expresión de un sistema social y de género profundamente desigual. La categoría feminicidio desmitifica la idea de violencia como asunto personal y muestra su carácter político, resultado de las relaciones desiguales de poder. Muestra que las mujeres son objeto de la violencia que se ejerce en un cuerpo biológico, en un cuerpo individual, pero también un cuerpo cultural atravesado por las relaciones económicas, raciales y de género, por la inseguridad y por un Estado que poco hace para evitar esas muertes. El feminicidio establece relaciones entre el nivel micro (las relaciones personales) y el nivel macro (el género y los otros 
determinantes sociales). Cuanto más autoritaria es una sociedad, más peligrosa es para las mujeres; cuanto mayor es la desigualdad, mayor el riesgo de feminicidio y de otras violencias. Asimismo, el riesgo no es igual para todas, hay grupos que están expuestos de forma desproporcionada a la violencia, porque están en situaciones íntimas o en relaciones sociales más peligrosas, o en ambas.

Los estudios muestran que el desempleo, la pobreza, la edad, el grupo étnico, la clase social, el aislamiento, la migración, los niveles de criminalidad del lugar en donde vive la mujer, la falta de recursos y de apoyo impactan en quién será más afectada, sufrirá violencia y estará en mayor riesgo de morir. Pero no se debe asociar la violencia con la pobreza, sino reconocer a las mujeres que están en mayor peligro que otras, para no caer en generalizaciones y hacer una mala ciencia y una mala política pública. Es necesario reconocer las situaciones particulares de vulnerabilidad en que se encuentran algunas mujeres y los factores de riesgo de feminicidio, incluyendo la historia de la cultura local, las desigualdades económicas, la impunidad en relación con los crímenes contra las mujeres, el sexismo y el racismo.

El feminicidio es un arma del patriarcado, del racismo, de la opresión económica, de la xenofobia, de la heteronormatividad y del coIonialismo; es una marca en los cuerpos que sufrieron múltiples formas de injusticias, resultado de un sistema de estratificación social en funcionamiento, de prácticas individuales del asesino, pero también de prácticas colectivas a las que he denominado "descartabilidad biopolítica de mujeres"(21). El feminicidio tiene un papel sistémico y fundamental que llega a establecerse como una necropolítica, un término desarrollado por el filósofo africano del siglo XX, Achille Mbembe, que habla de la violencia racista. El autor parte del concepto de biopoder elaborado por Michel Foucault ${ }^{(22)}$; sin embargo, lo considera insuficiente para explicar las formas contemporáneas de subyugación de la vida al poder de la muerte. Así, Mbembe ${ }^{(23)}$ construye el concepto de necropolítica, que constituye el poder soberano que va a dictar quién puede vivir y quién debe morir. Ejercitar la soberanía es ejercer control sobre la mortalidad y definir la vida como la implantación y manifestación de poder. La noción de necropolítica y necropoder explican las diversas maneras en que, en el mundo contemporáneo, hay interés en la destrucción máxima de personas y en la creación de "mundos de muerte", como el campo de refugiados y el apartheid. La necropolítica y el necropoder hacen referencia, entonces, a una forma particular de existencia social que pone a grupos enteros a vivir debajo de la línea de lo humano. La necropolítica produce la convergencia de la violencia racista, la violencia de clase y la violencia de género, lo que construye un sistema de estratificación de discursos y prácticas que generan una política letal, que hace que algunos cuerpos sean más susceptibles de ser descartados.

Rita: ¿Cuáles son los nuevos determinantes de la violencia contra las mujeres y de su muerte?

Betty: Responder esa pregunta exige afinar el análisis para no caer en el lugar común del patriarcado o el machismo. Es necesario ir más allá en el análisis, dado que los crímenes contra las mujeres suelen ser leídos como violencia intrafamiliar, violencia de pareja o crímenes pasionales. Desde nuestra experiencia como pueblo negro/afrocolombiano, la violencia contra las mujeres, incrementada en los últimos 20 años en las regiones de poblamiento afrodescendiente, ha venido elevándose de formas sin precedentes, en el marco de la implementación de las políticas de libre mercado y desarrollo en nuestros territorios. En ese sentido ubico dos aspectos relevantes para entender las violencias contra las mujeres, muchas de las cuales terminan en asesinato. El primero, son los procesos de expropiación territorial a los pueblos y comunidades $y$, el segundo, la transformación de la subjetividad de estos pueblos. Lo que planteo es que el asesinato de mujeres en territorios etnizados y racializados como los de pueblos negros, tanto en Colombia como en todo el continente americano y el Caribe, 
corresponde a un proceso de colonización (inacabada) de los territorios, los cuerpos y los imaginarios de comunidades negras e indígenas. A las mujeres se las asesina porque su sentido comunitario y sus prácticas ancestrales que tejen comunidad son un obstáculo al avance del neoliberalismo que requiere individuos aislados para el libre mercado. Quiero mencionar, aunque sea brevemente, que construir individuos es también el papel de la evangelización neopentecostal de la teología de la prosperidad y la guerra espiritual. Es por esto que han declarado una guerra sin cuartel contra las religiones de origen africano que han sido el sustento espiritual de las luchas cimarronas del pueblo afrodescendiente en el continente. A las mujeres se las asesina, también, porque, como dice Rita Segato ${ }^{(22)}$, estos crímenes son útiles al despliegue de una pedagogía de la crueldad: en el cuerpo mutilado, masacrado, mil veces asesinado de una mujer se enseña a la comunidad, a otras mujeres y a la sociedad toda que esa mujer se lo merecía, por lo que no merece nuestra solidaridad ni nuestra compasión ni nuestra empatía. Es una forma de enseñarnos a no sentir por el otro, a separarnos, a desarticularnos. Ese es el papel de la pedagogía de la crueldad ejercida en el cuerpo de las mujeres. Familiarizarnos, además, con las formas atroces de matar. A estas conclusiones nos ha llevado la pregunta: ¿qué es lo que está ocurriendo en nuestras sociedades que hace que los hombres maten más mujeres que en épocas anteriores, siendo que siempre hubo o ha habido machismo? El feminicidio es más que un crimen pasional. El feminicidio es un crimen de Estado porque involucra situaciones que son estructurales al sistema-mundo capitalista, racista y colonialista. Necesitamos tener una explicación para lo que ha sucedido en Brasil en los últimos quince años en los que los feminicidios se han disparado. Tenemos que preguntar qué es lo que está pasando en Colombia, en Guatemala, o en Honduras, país con el mayor índice de violencia en el mundo contra las mujeres. ¿Qué es lo que está pasando? Los análisis buscan entender lo que pasa en el contexto sociopolítico, económico y cultural: qué transformaciones se dieron para que se produzcan muertes de mujeres de forma masiva. Históricamente, en el pensamiento de la población negra del Pacífico colombiano no se concebía causar daños irremediables a otras personas, y hoy, en esa misma región, una gran cantidad de mujeres son asesinadas y desaparecidas. ¿Qué transformación ocurrió en la subjetividad de estos sujetos? Efectivamente, esos hombres hacen parte de una estructura patriarcal; no obstante, hay un patriarcado actual que produce asesinatos de mujeres de una manera sin precedentes, ¿por qué sucede esto? A pesar de las violencias históricas ejercidas contra las mujeres negras e indígenas en este continente, por la conquista y la colonización, las mujeres negras logramos sobreponernos a esas violencias y liderar la construcción de mundos que nos devolvieron la humanidad como pueblo afrodescendiente. Lo que está sucediendo desde hace treinta años en la región del Pacífico colombiano es la destrucción de esos mundos creados bajo el liderazgo de las mujeres negras. Esa destrucción efectúa sobre los hombres una castración simbólica, que los convierte en hombres que no pueden ya responder, dadas las transformaciones ontológicas generadas por el neoliberalismo y el neoconservatismo, a las exigencias culturales en relación con su masculinidad. Es así que su único lugar de poder es su casa y su mujer, ese es el único control que poseen y él no puede permitir perderlo porque no será "nadie". Los hombres se convierten así en un arma del sistema mundo colonial moderno racista patriarcal, y esto todavía no lo entienden ni la izquierda ni el movimiento social afrodescendiente.

\section{La acción política y el fortalecimiento de los lazos comunitarios como estrategia frente a la violencia y a la muerte de mujeres}

Aline: ¿Cuáles son las posibilidades de resistencia frente a esa realidad?

Montserrat: Los feminicidios son marcas que permiten leer la realidad de un país. En los 
feminicidios se concentran las exclusiones sociales, la precariedad de la vida, el racismo, el clasismo y el sexismo. Entonces, a partir del cuerpo de una mujer asesinada se puede distinguir la estructura social, la desigualdad social. Esto significa que, para enfrentar el feminicidio, hay que cambiar la estructura social, empezando por transformar el tejido social y las comunidades, para establecer nuevas formas de solidaridad y combatir el individualismo exacerbado. Hay muchos ejemplos oriundos de los pueblos originarios, de las poblaciones negras, de las poblaciones campesinas de cómo reconstruir el tejido social y establecer nuevas relaciones de solidaridad. La única forma de sobrevivir en este planeta es reconstruir las redes de relaciones sociales, empezando desde abajo hacia arriba.

Evidentemente, tenemos que garantizar también que haya una respuesta rápida ante los feminicidios. Sabemos que la familia, los vecinos, las personas cercanas son fundamentales para prevenir un feminicidio. Es necesario, entonces, trabajar con las familias, con las redes e instituciones locales para que puedan detectar los signos de peligro: una mujer maltratada por su pareja, una joven empezando a involucrarse con el tráfico. Tenemos que asegurar que haya una respuesta local adecuada y tenemos que transformar también el Estado, luchar contra el modelo neoliberal, contra el modelo patriarcal, contra el modelo racista y colonial. Si no luchamos contra esos modelos no vamos a poder transformar la realidad y pensar en otro mundo sin feminicidios y sin violencia contra las mujeres y contra las poblaciones históricamente excluidas.

Esta es una tarea monumental, pero el feminicidio es reflejo de esta estructura de exclusión social. Es necesario pensar en acciones de la vida cotidiana, asumir nuestras responsabilidades y no delegar a otras personas, pues si no tomamos la iniciativa y solo nos quedamos esperando que alguien lo haga, no sobreviviremos.

Stela: Compañeras, ¿cuáles son sus palabras finales?, ¿qué aprendimos y compartimos en estos dos días de reflexiones y conversaciones entre mujeres latinoamericanas, hermanadas en las luchas contra las violencias y el femigenocidio?

Rita: Vamos a empezar explicando qué son los femigenocidios según Rita Segato ${ }^{(24)}$, quien nos dice que el término femigenocidio es un concepto que debe ser utilizado para los crímenes sistemáticos e impersonales, que tienen por objetivo específico la destrucción de las mujeres, solo por ser mujeres, sin posibilidad de personalizar o individualizar ni el móvil de la autoría ni la relación entre perpetrador y víctima. De esta forma, introducimos la partícula "geno" para denominar aquellos feminicidios que se dirigen a la mujer como genus, es decir, como género, en condiciones de impersonalidad. Este carácter genérico, impersonal y sistemático es indispensable para aproximarlos al perfil de los crímenes de lesa humanidad y para incluirlos en el fuero internacional que se ocupa de los crímenes de lesa humanidad y genocidio. Ante este escenario desafiante, es necesaria la acción política y el compromiso de cada una/o de nosotras/os, lo que significa el fortalecimiento de las redes, la apertura de espacios para el ejercicio del habla, la escucha y la acogida de narrativas singulares de mujeres negras, indígenas, blancas, pobres, migrantes, jóvenes y ancianas, buscando identificar situaciones de riesgo y vulnerabilidad y responder a cada una de acuerdo con sus necesidades.

Aline: En la búsqueda de cambios de esta situación difícil que vivimos en nuestra sociedad, en especial para las mujeres, pienso que necesitamos, más que nunca, de acciones colectivas que contribuyan al cambio de esta brutal realidad que pudimos acompañar y discutir durante el Seminario Internacional Rutas Críticas 8. En este sentido, resulta necesaria la integración de fuerzas, en diferentes sitios de la red, con el objetivo de potenciar acciones para enfrentar las violencias y construir relaciones más igualitarias en nuestra sociedad. Por eso, necesitamos repensar el papel de la universidad, el trabajo en el territorio, y la actuación en la atención primaria de la salud cerca de las familias y de sus realidades. 
Stela: Sin olvidarnos que una de las maneras de enfrentamiento de los feminicidios pasa por la politización de esas muertes, identificando los reales determinantes que incluyen el capitalismo patriarcal, sexista, racista, xenófobo y colonial, como bien señalaron las compañeras. La acción política comprende no solo la capacidad de indignarnos, de organizarnos en colectivos, de denunciar estas muertes, sino también de rendir homenaje y reverencia a la memoria de las víctimas, de usar el arte como instrumento de elaboración del dolor y del duelo, en fin, de aliarnos con las fuerzas progresistas de la sociedad y con los excluidos de la historia, pensando que necesitaremos una vez más organizarnos para resistir.

\section{REFERENCIAS BIBLIOGRÁFICAS}

1. Meneghel SN, (org.). Rotas críticas: mulheres enfrentando às violências. São Leopoldo: UNISINOS; 2007.

2. Meneghel SN, (org.). Rotas críticas II: ferramentas para trabalhar com a violência de gênero. Santa Cruz do Sul: Editora da Universidade de Santa Cruz do Sul; 2009.

3. Meneghel SN, Vial SRM. Rotas Críticas: mulheres enfrentando as violências. Athenea Digital. 2008;14:199-213.

4. Meneghel SN. Situações limite decorrentes da violência de gênero. Athenea Digital. 2012;12(3):227-236.

5. Meneghel SN, Portella AP. Feminicídios: conceitos, tipos, cenários. Ciência \& Saúde Coletiva. 2017;22(9):3077-3086.

6. Meneghel SN, Lerma BRL. Feminicídios em grupos étnicos e racializados. Ciência \& Saúde Coletiva. 2017;22(1):117-122.

7. Meneghel SN, Bairros FS, Mueller B, Monteiro D, Oliveira LP, Collaziol ME. Rotas críticas de mulheres em situação de violência: depoimentos de mulheres e operadores sociais em Porto Alegre, Rio Grande do Sul, Brasil. Cadernos de Saúde Pública. 2011;27(4):743-752.

8. Brockmeier J, Harré R. Narrativa: problemas e promessas de um paradigma alternativo. Psicologia: Reflexão e Crítica. 2003;16(3):525-535.
9. Ochs E. Narrativa. En: Van Djik TA, (comp.). El discurso como estructura y proceso: estudios sobre discurso I; Una introducción multidisciplinaria. Barcelona: Gedisa editorial; 2003. p. 271-304.

10. Sagot Rodríguez M, Carcedo A. La ruta crítica de las mujeres afectadas por la violencia intrafamiliar en América Latina: estudios de caso en diez países [Internet]. Washington DC: Organización Panamericana de la Salud; Programa Mujer, Salud y Desarrollo; 1998 [citado 10 may 2018]. Disponible en: https://tinyurl.com/y7nteh4d.

11. Radford J, Russell DEH. Femicide: the politics of women killing. New York: Twayne Publishers; 1992.

12. Lagarde M. Por la vida y la libertad de las mujeres, fin al feminicidio [Internet]. Cimacnoticias; 2004 [citado 10 may 2018]. Disponible en: https:// tinyurl.com/y839nnbu.

13. Segato RL. La escritura en el cuerpo de las mujeres asesinadas en Ciudad Juárez: territorio, soberanía y crímenes de segundo estado. México DF: Universidad del Claustro de Sor Juana; 2006.

14. Meneghel SN, Rosa BAR, Ceccon RF, Hirakata VN, Danilevicz IM. Feminicídios: estudo em capitais e municípios de grande porte populacional. Ciência \& Saúde Coletiva. 2017;22(9):2963-2970.

15. Leites GT, Meneghel SN, Hirakata VN. Homicídios femininos no Rio Grande do Sul, Brasil. Revista Brasileira de Epidemiologia. 2014;17(3): 642-653. 
16. Meneghel SN, Hirakata VN. Femicídios: homicídios femininos no Brasil. Revista de Saúde Pública. 2011;45(3):564-574.

17. Margarites AF, Meneghel SN, Ceccon RF. Feminicídios na cidade de Porto Alegre: Quantos são? Quem são? Revista Brasileira de Epidemiologia. 2017;20(2):225-236.

18. Meneghel SN, Ceccon RF, Hesler LZ, Margarites AF, Rosa S, Vasconcelos VD. Femicídios: narrativas de crimes de gênero. Interface-Comunicação, Saúde, Educação. 2013;17(46):523-533.

19. Waiselfisz JJ. Mapa da violência 2015: Homicídios de mulheres no Brasil [Internet]. Brasília DF: OPAS/SPM; 2015 [citado 10 may 2018]. Disponible en: https://tinyurl.com/yccd8l8y.

20. Carneiro S. Mulheres negras e violência doméstica: decodificando os números. São Paulo: Geledés; 2017.
21. Sagot Rodríguez M. ¿Un mundo sin femicidios?: Las propuestas del feminismo para erradicar la violencia contra las mujeres. En: Sagot Rodríguez M, (coord.). Feminismos, pensamiento crítico y propuestas alternativas en América Latina. Buenos Aires: CLACSO; 2017. p. 61-78.

22. Foucault M. Em defesa da soiedade: curso no Collège de France, 1975-1976. São Paulo: Martins Fontes; 2005.

23. Mbembe A. Necropolítica. Revista Arte \& Ensaios. 2016;(32):122-151.

24. Segato RL. Femigenocidio y feminicidio: una propuesta de tipificación. Revista Herramienta. 2012;(49):1-7.

\section{FORMA DE CITAR}

Meneghel SN, Maciazeki-Gomes RC, Sagot M, Lozano Lerma BR, Bairros FS, Martins AB. Mujeres conversando con mujeres: un encuentro en el seminario Rutas Críticas 8. Salud Colectiva. 2019;15:e1871. doi: 10.18294/sc.2019.1871.

Recibido: 21 de mayo de 2018 | Versión final: 22 de agosto de 2018 | Aprobado: 18 de septiembre de 2018

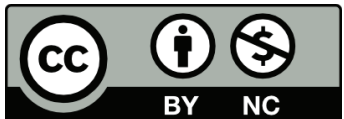

Esta obra está bajo una licencia de Creative Commons Reconocimiento-NoComercial 4.0 Internacional. Reconocimiento - Permite copiar, distribuir y comunicar públicamente la obra. A cambio, se debe reconocer y citar al autor original. No Comercial - Esta obra no puede ser utilizada con finalidades comerciales, a menos que se obtenga el permiso. 